\title{
Entrelacs
}

Cinéma et audiovisuel

Jodorowsky, d'un art à l'autre

\section{Le cinéma de Jodorowsky et la performance théâtrale : une « danse de la réalité »}

\section{Elisabeth Pouilly}

\section{(2) OpenEdition}

\section{Journals}

Édition électronique

URL : http://journals.openedition.org/entrelacs/4759

DOI : $10.4000 /$ entrelacs.4759

ISSN : 2261-5482

Éditeur

Éditions Téraèdre

Référence électronique

Elisabeth Pouilly, « Le cinéma de Jodorowsky et la performance théâtrale : une " danse de la réalité » », Entrelacs [En ligne], 16 | 2019, mis en ligne le 29 octobre 2019, consulté le 03 décembre 2019. URL http://journals.openedition.org/entrelacs/4759; DOI : 10.4000/entrelacs.4759

Ce document a été généré automatiquement le 3 décembre 2019

Tous droits réservés 


\title{
Le cinéma de Jodorowsky et la performance théâtrale : une « danse de la réalité »
}

\author{
Elisabeth Pouilly
}

\section{Introduction}

1 La Danza de la realidad, soit « la danse de la réalité », est un film d'Alejandro Jodorowsky sorti en 2013 et qui est l'adaptation d'une partie de son autobiographie éponyme. Cette expression nous semble caractéristique du rapport qu'entretient Jodorowsky avec « la réalité » : c'est une des notions clés de sa théorie théâtrale et il poursuit cette réflexion dans sa pratique cinématographique.

2 Si Jodorowsky est actuellement reconnu comme cinéaste, son théâtre est encore relativement méconnu. Son activité théâtrale débute par la découverte de l'art clownesque, des marionnettes puis du mime ${ }^{1}$. Mais il réalise aussi des « actes poétiques » dès sa jeunesse au Chili. Il utilise la rue et les lieux existants pour réaliser des actes devant faire éclater la logique apparente du réel et de la vie quotidienne : «Si nous regardions lucidement notre réalité, nous constaterions qu'elle est poétique, illogique, exubérante ${ }^{2}$.» Avec le poète Enrique Lihn, il décide un jour de marcher toujours en ligne droite, en montant sur les voitures ou en demandant aux personnes de traverser leur maison. Un autre acte poétique consiste à aller dans un grand restaurant et frotter plusieurs fois leur costume avec des steaks commandés :

Nous l'avons fait cinq ou six fois de suite jusqu'à ce que tout le restaurant bascule dans une sorte de panique. Un an plus tard, nous sommes revenus dans ce même établissement, et le maître d'hôtel nous a déclaré : « Si vous comptez recommencer ce que vous avez fait l'autre jour, pas question, nous ne vous acceptons pas. » L'acte l'avait tellement frappé que le temps s'en était trouvé comme stoppé. Une année s'était écoulée, mais il lui semblait nous avoir vus la semaine précédente ${ }^{3}$.

Les émotions ressenties transforment la perception du temps : l'acte artistique s'inscrit dans une durée variable et personnelle et non plus dans le temps réel. 
Jouer avec la réalité va amener Jodorowsky, après avoir mis en scène des pièces de théâtre d'auteurs contemporains (Fernando Arrabal, Eugène Ionesco, Samuel Beckett notamment) au Mexique, à créer des performances théâtrales qu'il appelle les éphémères paniques ${ }^{4}$, de 1962 à 1967. Ils ont lieu dans des endroits définis mais considérés comme " non théâtraux " : école d'art, piscine, cinéma, etc. L'idée est de bouleverser les habitudes des spectateurs:

Pour arriver à l'euphorie panique, il faut se libérer de l'édifice théâtre, et ceci avant tout. [...] La «bande» panique choisira le lieu qui lui plaira le plus: un terrain vague, un bois, une place publique, un amphithéâtre de chirurgie, une piscine, une maison en ruine, ou bien, un théâtre traditionnel, mais en employant tout son volume: manifestations euphoriques entre les fauteuils, dans les loges ou les toilettes, débordant le long des couloirs, dans la cave, le foyer, le toit, etc. On peut aussi faire un «éphémère » sous la mer, dans un avion, dans un train express, un cimetière, une maternité, un abattoir, un asile de vieillards, dans une grotte préhistorique, dans un bar d'homosexuels, un couvent, pendant une veillée mortuaire ${ }^{5}$.

5 Réalité et art se mêlent: le théâtre sort du théâtre pour s'intégrer aux lieux du quotidien. Jodorowsky permet ainsi aux spectateurs de voir leur réalité autrement puisqu'elle peut faire partie d'une œuvre d'art.

Ces expériences vont marquer l'ensemble de l'œuvre artistique de Jodorowsky, tant son théâtre que son cinéma ${ }^{6}$. La relation entre la performance théâtrale et le cinéma de Jodorowsky est ambiguë : la frontière en est poreuse. Si des performances peuvent être directement intégrées dans certains films, il s'agit surtout de voir quelles caractéristiques de la performance théâtrale passent dans le cinéma et dans quelle mesure cette forme d'art influence les longs métrages réalisés par Jodorowsky.

\section{Performance théâtrale et cinéma}

7 La performance théâtrale et le cinéma paraissent de prime abord opposés, la première étant l'art du présent, de l'éphémère et de la présence, quand le deuxième est un art de l'image s'inscrivant dans la durée, techniquement reproductible ${ }^{7}$.

8 Le terme de "performance théâtrale " s'entend ici comme une action performative, venant de l'art théâtral et mettant au cœur de sa problématique les caractéristiques spatio-temporelles du théâtre : l'ici et maintenant, au détriment du texte, de la création d'une fiction ou de personnages, et prenant en compte, d'une manière ou d'une autre, le spectateur. C'est ce que Christian Biet note comme étant l'essence du théâtre :

Le théatre est d'abord un spectacle, une performance éphémère, la prestation de comédiens devant des spectateurs qui regardent, un travail corporel, un exercice vocal et gestuel adressés, le plus souvent dans un lieu particulier et dans un décor particulier. En cela, il n'est pas nécessairement lié à un texte préalablement écrit, et ne donne pas nécessairement lieu à la publication d'un écrit ${ }^{8}$.

9 Jodorowsky, dans son texte théorique « Vers l'éphémère panique ou Sortir le théâtre du théâtre » écrit en octobre 1965, insiste particulièrement sur la caractéristique temporelle du théâtre :

En acceptant son caractère éphémère, le théâtre trouvera ce qui le distingue des autres arts - et par là même son essence. Les autres arts laissent des pages écrites, des enregistrements, des toiles peintes, des volumes objets-traces qui, avec le temps, finiront par s'effacer, mais par un processus très lent. Le théâtre, par contre, 
ne survivra même pas à un seul jour de la vie d'un homme. Au fur et à mesure qu'il naît, il devra mourir 9 . sources directes sur les éphémères paniques en dehors d'articles de journalistes. L'éphémère le mieux documenté est aussi le plus long: nommé Melodrama sacramental, littéralement en français « Mélodrame sacramentel », il a eu lieu à Paris le 24 mai 1965 et a duré plus de quatre heures. Il s'inscrit dans une soirée panique programmée dans le cadre du $2^{2 \mathrm{èe}}$ Festival de la Libre Expression dirigé par Jean-Jacques Lebel et organisé au Centre culturel américain de Paris. Cet événement est l'un des éphémères les mieux couverts par la presse. Jean-Jacques Humeau le filme et réalise un reportage vidéo intitulé He! Viva Dada ${ }^{10}$. Dans ce film, nous remarquons que la salle possède une scène sur laquelle se déroulent principalement les actions mais que les spectateurs en sont très proches. Tous les objets utilisés lors de l'éphémère (majoritairement des matières organiques, périssables) leur sont jetés ou donnés à la fin de la performance.

Il y a très peu de personnages: on peut seulement reconnaître un rabbin, et Jodorowsky, performeur principal de cette performance, se met en scène en impliquant totalement sa personne et son propre vécu. Il n'y a pas d'histoire dans le sens de fiction linéaire, mais plutôt des thèmes se succédant qu'on pourrait qualifier de mouvements car la musique change et accompagne les différentes actions. Le premier mouvement correspond à une transe collective amenée par une musique rock dominée par une batterie: Jodorowsky apparaît entièrement vêtu et casqué puis est dépouillé progressivement de tout ce qui peut le protéger ou le cacher, y compris sa chevelure. Le deuxième mouvement est amorcé par une plus grande place donnée à la guitare électrique combinée à la batterie : des croix sont amenées sur scène et plusieurs actes qui suivent peuvent être perçus comme une dénonciation du christianisme. Ensuite, une musique juive résonne et le personnage du rabbin entre. Il embrasse Jodorowsky sur la bouche puis en fait son pantin. Toutefois ce dernier finit par le repousser, il sort alors de la veste du rabbin des entrailles qu'il cloue sur une croix à laquelle il attache ensuite le chef religieux : le judaïsme est à son tour rejeté. Le dernier mouvement est marqué par une reprise de la batterie à certains moments au-dessus de la musique plus douce : Jodorowsky met en scène sa nouvelle naissance, après ce passage à la scène voulu comme une immolation de son être antérieur.

prermance se caractérise par la mise en présence simultanée des acteurs et des spectateurs et par une part laissée au hasard et aux interactions entre les différents protagonistes. Toutefois, les éphémères paniques ne sont pas totalement improvisés. La trame des actes réalisés dans la performance a été prévue à l'avance, et une fois sur scène les performeurs s'en emparent selon les circonstances et les inspirations du moment :

Ce spectacle panique avait été minutieusement préparé. J'avais acquis une expérience et n'avançais plus à l'aveuglette: les risques étaient pris en toute connaissance de cause. Montant cet événement, j'étais conscient d'aller au-devant d'une mort, d'un rite de passage dont je ne pourrais sortir qu'anéanti ou transformé...11

13 L'implication émotionnelle et le danger physique semblent plus élevés pour le performeur, qui est au centre du dispositif théâtral, que pour l'acteur de cinéma, qui est un des éléments captés par la caméra. Un espace peut devenir un lieu théâtral dès qu'un acteur y évolue tandis que le cinéma n'existerait pas sans ses dispositifs : la caméra, la pellicule de celluloïd ou la carte mémoire actuellement et le projecteur. 
L'acteur y est presque secondaire: un espace devient cinématographique s'il est simplement capté par une caméra tandis que le théatre et encore plus la performance ressentent la nécessité du corps de l'acteur. La réalité de ce corps au théâtre est accréditée par le hic et nunc partagé avec le spectateur, quand au cinéma, l'image révèle avec intensité une réalité passée ${ }^{12}$. Le processus technique du cinéma impose une réalité paradoxale des corps perçus : ils n'existent pas dans la même temporalité que ceux des spectateurs mais continuent à vivre devant leurs yeux. André Bazin indique que dans l'art photographique, et il en va de même pour le cinéma, l'homme est certes exclu mais « nous jouissons de son absence ${ }^{13}$ ».

14 Cette présence actuelle ou différée change dans ces arts le rapport aux spectateurs. En effet, la perception de la réalité n'est pas la même pour le spectateur de théâtre, qui plus est de performance, et celui de cinéma. Joseph Danan déclare ainsi, dans son essai Entre théâtre et performance: la question du texte, que dans la performance il n'y a "pas d"“effet de réel", comme celui que peut produire le cinéma, mais un "effet réel" ${ }^{14}$ ". Christian Metz décrivait « l'impression de réalité $»^{15}$ propre au cinéma, qui caractérise l'immersion du spectateur dans un film et une adhésion au système diégétique présenté par celui-ci. Cette impression de réalité est la même que celle produite par le rêve. Roland Barthes pose quant à lui l'« effet de réel $»^{16}$ d'une œuvre comme une construction rapprochant la fiction de l'expérience du réel acquise par les spectateurs. Cette ressemblance apportée par des détails ne renforce pas seulement la croyance du spectateur en la fiction représentée mais brouille un instant la ligne de démarcation entre réel et fiction, jetant un trouble dans son esprit :

L'effet de réel, comme le syntagme l'indique, n'est pas une impression (au sens d'une immersion) mais bien un effet (au sens d'un trouble) dont la dimension de prise sur le récepteur est essentielle ${ }^{17}$.

Quand Joseph Danan parle d'« effet réel ", le trouble du spectateur n'est plus lié à la reconnaissance d'une situation possible dans le réel mais à la contamination de la fiction par le réel. La limite entre les deux est poreuse : sommes-nous encore dans une création artistique cherchant le vraisemblable ou bien dans un acte réel à volonté artistique ? Le spectateur face à cette incertitude ressent alors un malaise car l'œuvre dépasse les limites habituellement conférées à l'art et peut avoir des conséquences dans la vie réelle (notamment celle de l'artiste). Les actes artistiques prennent soudain par « l'effet réel » le même poids, la même densité que « les faits réels ».

Le cas de la performance entendue dans le domaine des arts plastiques est une belle illustration de cette affirmation. Par exemple, quand Chris Burden se fait volontairement tirer dessus par un ami dans une galerie californienne en $1971^{18}$, le risque pris est réel et sa blessure au bras est tangible et vraie ${ }^{19}$. Dans la performance théâtrale Melodrama sacramental, Jodorowsky se fait réellement fouetter sur scène par une femme. Ce qu'il reste de l'acte est inscrit dans son corps : il devient le premier témoin durable de l'acte artistique ayant eu lieu, dont la trace perdure un temps par ce biais avant de s'effacer.

Cette implication du corps de l'acteur est généralement moindre au cinéma car on peut avoir recours à des effets spéciaux, des doublures, des cascadeurs, etc. La réalité de ce qui est vu peut être mise en doute, le cinéma apparaissant comme un art de l'artificialité, presque du mensonge, tant sa technique offre la possibilité de faire croire, durant un temps limité, à tout un monde imaginaire. Pourtant, Jodorowsky tourne ses 
films de manière à approcher de la réalité et des conditions de la performance théâtrale.

\section{Un cinéma proche des conditions de la performance}

Fando et Lis (Fando y Lis), le premier long métrage de Jodorowsky sorti en 1968, est une adaptation libre de la pièce de théâtre éponyme de Fernando Arrabal publiée en 1957. Auparavant, en 1961 et 1967, Jodorowsky a mis en scène la pièce dans plusieurs théâtres de Mexico en suivant le texte d'Arrabal et en en proposant deux univers très différents : le premier très oppressant (la scène est majoritairement occupée par des murs faits de planches en bois disjointes) et le deuxième très sobre mais sans point de repère. Jodorowsky tourne son film dans un lieu désertique qui évoque à la fois la quête philosophique des personnages et leur errance physique. Un seul lieu fermé apparaît dans le film : une pièce dans laquelle les deux acteurs principaux peignent leurs noms sur leurs corps et sur les murs. Nous pouvons parler d'intégration d'une performance au sein du film : le corps des acteurs devient lui-même une œuvre artistique, plastique, et le centre de cette performance. De plus, le spectateur de cinéma, ordinairement déjà en position de voyeur, ressent cette position plus fortement car la caméra est portée. Ainsi, il entre par le biais du caméraman dans l'espace clos où se déroule l'acte performatif. La présence sentie du corps du technicien, par le mouvement qu'il imprime à la caméra, et son absence sur l'image permettent de donner une « corporalité » à l'outil enregistreur : le spectateur n'est plus seulement un œil mais un corps, celui du cadreur. Ce passage de Fando et Lis est aussi différent du reste du film car il ne s'inscrit plus dans la logique diégétique : certes, les acteurs s'écrivent encore les prénoms des personnages sur le corps, mais Lis marche dans cette séquence alors que dans tout le reste du film, elle est handicapée et ne peut pas bouger ses jambes. De plus, Jodorowsky indique ${ }^{20}$ que la performance a pris plus d'ampleur que prévu car les acteurs en seraient venus d'eux-mêmes à projeter l'encre sur les parois et sur leurs corps. Même si la parole du réalisateur peut être sujette à caution, la séquence montre toutefois une volonté de la part de Jodorowsky de se rapprocher du réel et de réintégrer le hasard, l'imprévu, ou son simulacre, dans le film.

D'autres performances intégrées à des films de Jodorowsky sont caractérisées moins par la part laissée au hasard que par l'« effet réel » que le spectateur perçoit. Dans La Montagne sacrée (La Montaña sagrada, 1973), Jodorowsky met en scène une séquence dans laquelle explosent des crapauds et des caméléons déguisés soit en indiens soit en conquistadors. À la parodie joyeuse et théâtralisée de la conquête du Mexique par les Espagnols s'oppose soudain la réalité de la mort des animaux.

Moins visible, dans Fando et Lis, le personnage du "voleur de sang » de Lis est joué par un vrai médecin volontaire. L'« effet réel» ressenti ici tient au fait que l'action consistant à faire une prise de sang à Lis puis à boire celui-ci est vraiment réalisée par l'actrice et l'émotion qui se lit sur son visage n'est pas feinte. La séquence est par ailleurs à mettre en parallèle avec un éphémère qui a eu lieu au théâtre Jesús Urueta à Mexico où un médecin était venu sur scène faire une prise de sang aux actrices avant que celles-ci n'obligent Jodorowsky à le boire ou plutôt à manger le sang coagulé.

Pourquoi au cinéma avoir ce souci de la réalité quand à l'écran la différence risque de passer inaperçue ? Le film Santa Sangre sorti en 1989 permet de saisir que l'authenticité de l'émotion prime sur le souci de réel. Dans celui-ci, un jeune garçon joué par Adam, le 
fils cadet de Jodorowsky, se fait tatouer au couteau un immense oiseau sur la poitrine. Jodorowsky décide de cadrer en gros plan le visage du garçon qui crie et pleure, pendant un temps assez long, ce qui génère un certain malaise chez le spectateur. Dans plusieurs entretiens, il explique qu'après en avoir parlé à Adam et avoir reçu son accord, il s'est posté sous la caméra et l'a pincé : même si l'action suggérée par la fiction n'est pas réelle, l'émotion éprouvée par le garçon et captée par la caméra est vraie et peut se transmettre aux spectateurs. Dans les commentaires audio de Santa Sangre, Jodorowsky s'entretient avec Alan Jones et revient sur le rejet du "théâtre » dans le sens de l'artifice et d'un apprentissage technique :

- [A. Jones] Vous le pincez pendant toute la scène.

- [A. Jodorowsky] Oui, mais ce qui compte c'est le résultat. Non? On a le gros plan

de quelqu'un qui vit quelque chose et ce n'est pas truqué [« this is not theatre »] ${ }^{21}$.

Le fait de privilégier le choix d'acteurs non professionnels, voire parfois jouant leur propre rôle, renforce aussi la véracité des phénomènes captés par la caméra. Dans Santa Sangre, Jodorowsky tourne beaucoup de plans autour de la place Garibaldi de Mexico et fait appel aux habitants comme figurants: les mariachis, les prostituées, etc. Il n'informe pas les passants de la signification de la scène à laquelle ils assistent lors de la marche funèbre pour la mort de l'éléphant, guettant leur réaction spontanée face à l'immense cercueil. L'intérêt qu'il porte aux groupes de figurants, qui deviennent parfois des foules entières dans ses derniers films, montre aussi une volonté d'intégrer une performance collective. Deux séquences s'inscrivent dans cette catégorie dans Poesía sin fin (2016) : celle du carnaval, où une centaine de personnes déguisées en squelettes et en diables (dont certaines font partie d'un véritable groupe de carnaval) défilent dans les rues; et celle du chapiteau de cirque où le public est composé de figurants ayant répondu à un appel sur Internet. Il soutient l'acteur Adam Jodorowsky exécutant un slam ${ }^{22}$ dans les gradins.

Le film qui va le plus loin dans cette volonté d'approcher la performance théâtrale est La Montagne sacrée. Si la première partie de ce film est dominée par des espaces clos et une mise en scène très formaliste, la dernière est quasiment entièrement tournée en extérieur et prend des allures de cinéma documentaire. Dans tous ses films, Jodorowsky fait le choix de tourner beaucoup de scènes en extérieur et en décors naturels ${ }^{23}$. Toutefois, dans le cas de La Montagne sacrée, le désir de faire éprouver des émotions authentiques aux acteurs passe aussi par la préparation du film en amont. L'œuvre d'art devient ainsi le prolongement d'une expérience communautaire et le thème initiatique du film est doublé par une véritable volonté d'initier les acteurs à un dépassement de soi :

La préparation a été très longue: je les ai enfermés pendant deux mois, pour accomplir quatre fois par jour des exercices. [...] Des disciples d'Ichazo, un homme et une femme, sont venus donner des leçons. On dormait quatre heures par jour. On se couchait à minuit, pour se faire réveiller à quatre heures du matin en faisant des exercices d'éveil, une douche froide, et toute la journée se passait en exercices, avec une diète spéciale ${ }^{24}$.

La façon de filmer de Jodorowsky sert aussi cette recherche de réactions et d'émotions vraies chez les acteurs: il tourne rapidement et dans l'ordre chronologique. Il s'exprime sur ce point pour Santa Sangre mais emploie cette méthode pour tous ses films :

- [Alan Jones] Vous avez tourné dans l'ordre, comme à votre habitude.

- [Alejandro Jodorowsky] Autant que j'ai pu, oui.

- Pourquoi faites-vous ça? 
- Parce que les acteurs évoluent avec le film. Je tourne dans l'ordre pour que l'acteur ait le sens de la réalité. C'est aussi valable pour moi. Je sais que ça coûte plus cher, mais c'est mieux. [...] ça a été très bien fait et tourné rapidement. Je ne fais qu'une ou deux prises ${ }^{25}$.

\section{cinématographique. \\ Contre l'illusion théâtrale et l'illusion cinématographique} L'action unique "panique » trouve sa place au cinéma. En jouant sur l'« effet réel», sentiment du spectateur que l'acte artistique représenté est proche de la vie réelle, Jodorowsky engage a contrario une réflexion sur l'illusion, tant théâtrale que

Théâtraliser, c'est " en rajouter »- sans nuance péjorative. Agir sur l'artifice, et contrairement à un cinéma qui veut à tout instant nous en faire accroire, c'est conserver la conscience du jeu, des rideaux, de la salle qui écoute ${ }^{26}$.

Les éphémères de Jodorowsky se posent contre cette « théâtralisation » qui serait l'art d'« en rajouter » mais joue avec la présence des spectateurs dans la salle, avec cette tension qui s'établit entre le jeu et la vie, et entre deux espaces de la salle apparemment bien définis. La scène présente un espace de vie rempli d'objets périssables et dans lequel les acteurs évoluent en pouvant improviser à partir d'une trame définie. L'espace consacré au public est aussi pris en compte dans la performance théâtrale, même si les actions principales ne s'y situent pas : aux actions des acteurs doivent répondre les réactions du public. Pour amplifier ce phénomène, Jodorowsky entre parfois directement en communication avec les spectateurs, en les interpellant, en les provoquant, ou encore en leur jetant des objets. Lors de l'éphémère à l'école d'art San Carlos de Mexico le 23 octobre 1963, il lance des bandes dessinées aux étudiants en Arts plastiques en leur criant : «Oui, prenez, lisez. Ceci est la seule chose que vous comprenez ${ }^{27}$. »

Dans une réflexion plus théorique, il brouille les normes et les connaissances de ces normes par les spectateurs. Il annonce ainsi la fin possible de cet éphémère, tout en disant qu'il ne sait pas vraiment quand commence et quand finit un éphémère, laissant alors le public libre d'agir : «L'auditoire ne savait pas s'il fallait applaudir, et dans un étrange brouhaha, mêlé d'applaudissements, il a commencé à défiler vers la sortie $[\ldots]^{28} . »$

28 Dans son cinéma, Jodorowsky bouscule aussi l'horizon d'attente du spectateur : son cinéma ne veut pas « en faire accroire ». L'« effet de réel » du cinéma de Jodorowsky ne tient pas dans l'utilisation discrète d'artifices mais dans la mise en lumière de ceux-ci. Par exemple, dans La Montagne sacrée le massacre des manifestants sur la planète Mars n'est pas montré de manière réaliste: des fruits tels que des fraises et des pastèques sont utilisés pour leur couleur à la place du sang et l'armée lance sur la foule des seaux de liquide rouge, mais celui-ci peut aussi être bleu, ou encore sortir clairement de tuyaux. À la fin de la séquence, certains manifestants bougent encore alors qu'ils sont censés être morts. Cela rend le passage plus supportable, plus poétique et le spectateur devient complice, non du massacre, mais des trucages.

Une autre manière de se rapprocher du spectateur est de s'adresser directement à lui par le biais de la caméra. Dans La Danza de la realidad, film adapté d'un passage du roman autobiographique éponyme ${ }^{29}$, Jodorowsky intervient directement à l'âge où il 
tourne, s'adressant soit à l'enfant qu'il était soit au spectateur. Trois passages face caméra sont remarquables : le premier implique le spectateur dans le choix qu'il est demandé au jeune garçon de faire entre ses croyances et les dires de son père; le deuxième est un commentaire par Jodorowsky d'une scène de son enfance qui se rejoue sous ses yeux $^{30}$; le troisième est la dernière scène $\mathrm{du}$ film dans laquelle Jodorowsky octogénaire prend la place de l'enfant qu'il était et opère un retour au présent, voire anticipe sur sa mort en se cachant derrière un personnage de squelette.

Au début de Poesía sin fin, Jodorowsky apparaît aussi tel qu'il est aujourd'hui, dans la rue de son enfance ayant subi des détériorations liées au temps et des changements sociologiques. Ce face à face avec le spectateur est suivi d'une mise en lumière du travail des techniciens. Ceux-ci apparaissent vêtus entièrement de noir et cagoulés, exactement comme peuvent l'être les marionnettistes du théâtre japonais de type bunraku, à la fois visibles et secondaires. Ils mettent en place le décor, notamment en tirant des toiles devant les habitations, ou encore en faisant avancer le train peint sur des panneaux, en deux dimensions. On assiste alors pour la mise en place de la fiction à un véritable "changement de décor" théâtral, mais sans la présence du rideau ou l'extinction des lumières, tout se passant devant la caméra. L'apparition de ces éléments théâtraux brise les habitudes du spectateur de cinéma par un mélange des genres.

31 Un travail inverse sur le temps, avec le retour à la fin du film au présent du tournage, est réalisé dans La Montagne sacrée, où cela va jusqu'à dévoiler le plateau et l'équipe de techniciens au travail. Le spectateur est interpellé face caméra par le personnage de l'alchimiste qui ne se dépouille plus seulement de son habit de sage mais se révèle comme le réalisateur donnant l'ordre au cadreur d'effectuer un zoom arrière. La révélation du processus de création du film rompt avec la fiction et va à l'encontre de l'attente $d u$ spectateur. Ce faisant, le temps du tournage et celui du visionnage semblent se rejoindre. La fiction a abouti au présent de sa production et Jodorowsky engage les spectateurs à revenir aussi à leur présent. Le film est un modèle pour le spectateur : celui-ci ne doit pas se projeter dans les personnages comme s'il croyait à la fiction qui lui a été proposée mais il doit effectuer sa propre quête de soi et trouver sa vérité derrière le masque du quotidien.

\section{Conclusion}

En 1965, Jodorowsky résumait dans une affirmation provocatrice le but du théâtre : « Si le but des autres arts est de créer des œuvres, celui du Théâtre sera de changer l'homme directement: le Théâtre n'est pas un Art mais une Science de la vie ${ }^{31} »$. La pratique est beaucoup moins caricaturale et indique plutôt une volonté constante de jouer avec la frontière entre l'art et la vie, dans une "danse de la réalité" ininterrompue. Cela se remarque tant dans ses performances théâtrales que dans son cinéma : si Jodorowsky tente d'intégrer une part de hasard dans ses productions en les rapprochant ainsi de la vie quotidienne, il travaille aussi sur la mise en lumière de ce qui est proprement artistique.

Dans son dernier film à visée documentaire intitulé Psychomagie, un art qui guérit (2019), il mêle encore l'aspect théâtral et cinématographique en revenant sur une pratique qu'il veut thérapeutique. Toutefois, même avec ce but, il insiste sur la nature artistique de la psychomagie dans la description de son projet: 
Nous suivons les sujets tout au long de leur processus de guérison, depuis leur premier témoignage, puis la réalisation de leur acte, puis enfin leur conclusion et ce que l'acte aura produit en eux quelques temps plus tard.

Les actes de Psychomagie doivent être impressionnants pour marquer l'inconscient et être efficaces, ils sont de fait hautement cinégéniques.

Avec le film Psychomagie, un art qui guérit, Alejandro filme la réalité mais une réalité intensifiée, magique et guérisseuse, sans acteurs mais avec de vraies personnes qui expriment leur souffrance et leur volonté d'en sortir ${ }^{32}$.

\section{" réalité ». Ses œuvres sont un appel à faire l'expérience personnelle du dépassement} de ces limites.

\section{NOTES}

1. Il écrit notamment La Cage et Le Mangeur de cœur pour le mime Marceau entre 1955 et 1960.

2. Alexandro Jodorowsky, Le Théâtre de la guérison, Paris, Albin Michel, 1995, p. 42.

3. Ibid., p. 39-40.

4. En 1962, à Paris, Alejandro Jodorowsky, Roland Topor et Fernando Arrabal créent le groupe Panique, dénomination que Jodorowsky garde pour désigner ses éphémères.

5. Alexandro Jodorowsky, "Vers l'éphémère panique ou Sortir le théâtre du théâtre " dans Fernando Arrabal, Panique : manifeste pour le troisième millénaire, Paris, Punctum, 2006, p. 88-89.

6. Jodorowsky poursuit ensuite sa création théâtrale par un théâtre plus écrit, qui a mis plusieurs années avant d'être représenté ; nous pouvons citer la pièce L'École des ventriloques, montée au Festival off d'Avignon par la Compagnie Point Zéro en 2014 ou encore celle intitulée Le Gorille, interprétée par Brontis Jodorowsky et jouée à Paris au Lucernaire entre 2010 et 2011.

7. Voir Walter Benjamin, L'CEuvre d'art à l'époque de sa reproductibilité technique [1936], Paris, Allia, 2011.

8. Christian Biet et Christophe Triau, Qu'est-ce que le théâtre ?, Gallimard, " Folio essais », 2006, p. 7.

9. Alexandro Jodorowsky, "Vers l'éphémère panique ou Sortir le théâtre du théâtre " dans Fernando Arrabal, Panique : manifeste pour le troisième millénaire, op. cit., p. 82.

10. Les bobines de ce reportage ont longtemps été considérées comme perdues avant de réapparaître et d'être achetées par le Centre Pompidou de Paris en 2009.

11. Alexandro Jodorowsky, Le Théâtre de la guérison, op. cit., p. 65.

12. Roland Barthes nomme ce sentiment de reconnaissance d'une réalité passée le «punctum » (voir La Chambre claire, Paris, Gallimard, 1980).

13. André Bazin, «Ontologie de l'image photographique », dans Qu'est-ce que le cinéma? [1958], Paris, Les Éditions du Cerf, coll. « 7e art », 1985, p. 13.

14. Joseph Danan, Entre théâtre et performance : la question du texte, Paris, Actes Sud-Papiers, 2013, p. 19.

15. Christian Metz, "À propos de l'impression de réalité au cinéma », dans Cahiers du cinéma, n¹66-167, mai-juin 1965, p. 74-83.

16. Roland Barthes, «L'effet de réel » dans Communications, $n^{\circ} 11,1968$, p. 84-89.

17. Hervé Glevarec, "Trouble dans la fiction. Effets de réel dans les séries télévisées contemporaines et post-télévision », dans Questions de communication, n 18, 2010, p. 221. 
18. Il s'agit de la performance intitulée Shoot.

19. Cette performance est citée en exemple par Joseph Danan, dans Entre théâtre et performance : la question du texte, op.cit., p. 18-19.

20. Commentaires dans Alejandro Jodorwsky, Fando et Lis [DVD], Paris, Wild Side Video, 2007, 1'00'03.

21. Commentaires dans Alejandro Jodorowsky, Santa Sangre [DVD], Paris, Wild Side Video, 2008, 28'44. La traduction est celle des sous-titres. Nous avons néanmoins rétabli entre crochets l'original anglais lorsque le sens était sensiblement différent de la traduction ou lorsque l'original apportait une nuance essentielle qui ne se retrouve pas dans la traduction.

22. Le " slam " dont il est question vient de l'univers des concerts rock et consiste à se faire porter à bout de bras par le public et à se déplacer ainsi au-dessus de lui.

23. Même dans La Danza de la realidad, s'il reconstitue les façades des maisons comme dans son souvenir, Jodorowsky le fait dans son village natal au lieu de le faire en studio.

24. Jean-Paul Coillard, De la Cage au grand écran, entretien avec Alejandro Jodorowsky, Paris, K-Inite, 2009, p. 200-201.

25. Commentaires dans Alejandro Jodorowsky, Santa Sangre [DVD], op. cit., 1'42'46.

26. René Gardies, Glauber Rocha, Paris, Seghers, coll. «Cinéma d'aujourd'hui », 1974, p. 94, cité dans Michel Larouche, Alexandre Jodorowsky cinéaste panique, Paris, Albatros, 1985, p. 63.

27. Lettre de « Rafael » reproduite dans Alejandro Jodorowsky, Teatro pánico, México, Era, 1965, p. 77 : « Sí, tomen, lean. Esto es lo único que entienden. » La traduction en français des extraits de cette lettre est celle de l'auteur de l'article.

28. Ibid., p. 79 : «El auditorio no supo si aplaudir o qué, y en un extraño griterío, mezclado con aplausos, empezó a desfilar hacia la salida [...]. »

29. Alejandro Jodorowsky, La Danse de la réalité, Paris, Albin Michel, 2001.

30. Il fait le lien alors entre la fiction et le spectateur car il est à la fois lui-même spectateur de la scène et acteur au sein de son film.

31. Alejandro Jodorowsky, "Vers l'éphémère panique ou Sortir le théâtre du théâtre ", dans Fernando Arrabal, Panique : manifeste pour le troisième millénaire, op. cit., p. 82.

32. Description du projet sur le site de financement participatif Ulule : https://fr.ulule.com/ jodorowsky/ (consulté le 03/10/2018), souligné dans le texte. Le présent article a été écrit un an avant la sortie du film, prévue en octobre 2019, et s'appuie donc sur le projet qui en avait été présenté à l'époque (NDLR).

\section{RÉSUMÉS}

Alejandro Jodorowsky a débuté ses performances théâtrales appelées " éphémères paniques » au Mexique en 1962 et les a poursuivies jusqu'en 1967. Cette forme d'art a influencé dans une certaine mesure ses longs métrages. La performance théâtrale et le cinéma paraissent au premier abord opposés par leurs caractéristiques techniques et dans le rapport au temps et au public qu'ils entretiennent. Art du présent, la performance théâtrale se veut plus proche du " réel » quand le cinéma est un art de l'image et a priori de l'illusion. Toutefois, Jodorowsky tourne ses films de manière à approcher les conditions de la performance théâtrale et tente d'y intégrer une part de hasard. Dans son cinéma, il engage aussi une réflexion sur l'illusion. Il met en lumière les trucages, engage le spectateur à un recul critique et parfois à un retour à sa réalité du moment. 
De même que les performances théâtrales de Jodorowsky luttent contre l'illusion théâtrale, ses films se posent contre l'illusion cinématographique et jouent avec les limites de cet art.

Alejandro Jodorowsky began his theatrical performances called " panic ephemera " in Mexico in 1962 and continued them until 1967. This type of art influenced to a certain extent his feature films. The theatrical performance and the cinema seem at first sight opposed by their technical characteristics and in relation to the time and the public that they maintain. Art of the present, the theatrical performance is closer to the "real" when the cinema is an art of the image and a priori of the illusion. However, Jodorowsky turns his films so as to approach the conditions of the theatrical performance and tries to integrate a part of chance. In his cinema, he also engages a reflection on the illusion. It highlights special effects, engages the viewer to a critical retreat and sometimes to a return to its reality of the moment. Just as Jodorowsky's theatrical performances fight against the theatrical illusion, his films arise against the cinematographic illusion and play with the limits of this art.

\section{INDEX}

Mots-clés : Alejandro Jodorowsky, éphémères paniques, performance théâtrale, illusion théâtrale, illusion cinématographique

Keywords : Alejandro Jodorowsky, panic ephemerals, theatrical performance, theatrical illusion, cinematic illusion

\section{AUTEUR}

\section{ELISABETH POUILLY}

Elisabeth Pouilly est docteure en Études Théâtrales. Elle a soutenu sa thèse sur «L" état d'esprit performatif" dans le théâtre et le cinéma d'Alejandro Jodorowsky » à l'Université Paris III Sorbonne Nouvelle, sous la direction de Joseph Danan. Elle a publié des articles sur les éphémères paniques, sur les liens entre le groupe Panique et Dada, sur les Midnight movies, et un article dans la revue d'art contemporain Marges traitant de l'expérience psychomagique de Jodorowsky. 